\title{
La responsabilidad social universitaria y la salud mental del personal administrativo, docente y estudiantes de la Universidad Nacional Jorge Basadre Grohmann de Tacna
}

\author{
University social responsibility and mental health of \\ administrative staff, professors and students at Jorge Basadre \\ Grohmann National University in Tacna
}

\author{
Victoria Nora Vela de Córdova ${ }^{1 a}$ \\ María Soledad Porras Roque ${ }^{1 \mathrm{~b}}$
}

${ }^{1}$ Escuela Académico Profesional de Enfermería de la Universidad Nacional Jorge Basadre Grohmann. Tacna, Perú.

${ }^{a}$ Docente, Doctor en Enfermería.

${ }^{\mathrm{b}}$ Docente, Doctora en Ciencias: Enfermería.

\section{RESUMEN}

Objetivo: Este estudio tiene como objetivo determinar la responsabilidad social universitaria y la salud mental positiva del personal administrativo, docentes y estudiantes como actores educativos de la Universidad Nacional Jorge Basadre Grohmann en el año 2018. Materiales y métodos: Es un estudio descriptivo, transversal, cuya muestra estuvo constituida por 636 actores educativos: 348 estudiantes, 162 docentes y 126 administrativos, utilizándose un muestreo no probabilístico por conveniencia. Los instrumentos que se utilizaron fueron dos: el cuestionario de responsabilidad social y el cuestionario de salud mental positiva, los cuales fueron usados para docentes, estudiantes y administrativos. La información fue recolectada y procesada con SPSS 22. Resultados: El 47,6\% de los administrativos manifestaron que existía una política explicita para no segregar el exceso a la formación académica de la población indígena, minoría racial, estudiantes de escasos recursos etc. en la universidad. Respecto a la salud mental positiva el $81,5 \%$ de los administrativos y docentes, en la subdimensión de habilidades cognitivas y emotivas de imaginar que se forma parte de la sociedad y de cómo se sienten las personas, manifestaron siempre asumir la responsabilidad de sus actos y decisiones. Conclusiones. Existe diferencia significativa en la concepción de la responsabilidad social universitaria del personal administrativo, docentes y estudiantes como actores educativos. Existe diferencia significativa en la concepción de la salud mental positiva de los actores educativos.

Palabras clave: Responsabilidad social universitaria, salud mental, salud mental positiva.

\section{ABSTRACT}

Objective: To determine the university social responsibility and positive mental health of administrative staff, professors and students as educational actors at the Jorge Basadre Grohmann National University in 2018. Materials and methods: It is a descriptive, cross-sectional study, whose sample consisted of 636 educational actors: 348 students, 162 professors and 126 administrators, using a non-probabilistic sample for convenience. The instruments were two: the social responsibility questionnaire and the positive mental health questionnaire, which were used for professors, students and administrators. The information was collected and processed with SPSS 22. Results: $47.6 \%$ of the administrators stated that there was an explicit policy in the university so as not to segregate the access to university education to indigenous population, racial minority, lowincome students etc. at Jorge Basadre Grohmann National University. Regarding positive mental health, $81.5 \%$ of administrators and professors, in the sub-dimension of cognitive and emotional skills of imagining that they are part of society and how people feel, claimed that they always take responsibility for their actions and decisions. Conclusions: There is a significant difference in the conception of university social responsibility of administrative staff, professors and students as educational actors. There is a significant difference in the conception of positive mental health of educational actors.

Keywords: University social responsibility, mental health, positive mental health. 


\section{Introducción}

Nos encontramos en una era de constante cambio y en una sociedad cada vez más dependiente a las nuevas tecnologías. Se ha llegado a un punto donde las fronteras geográficas han perdido relevancia, dado que la globalización ha generado una comunidad global a través de las tecnologías de comunicación. Así, este nuevo panorama otorga especial importancia a la identificación de las fortalezas y/o debilidades del grupo humano para el trabajo colaborativo.

Una de las fortalezas en potenciar en el recurso humano es la responsabilidad social. La universidad, según Ley Nº 30220 (1), se encuentra comprometida con ese aspecto y destaca la gestión ética y eficaz del impacto generado por la universidad en la sociedad debido al ejercicio de sus funciones académicas, de investigación y de servicios de extensión y participación en el desarrollo nacional, local y regional en sus diferentes niveles y dimensiones. También, incluye la gestión del impacto producido por las relaciones entre los miembros de la comunidad universitaria, sobre el ambiente, y sobre otras organizaciones públicas y privadas que se constituyen en partes interesadas.

Asimismo, los vertiginosos mecanismos de las sociedades contemporáneas traen nuevas exigencias a las personas afectando la salud mental; según la OMS en estos últimos años los trastornos mentales representan aproximadamente el $12 \%$ de la carga total de las enfermedades. En el Perú, el MINSA informa que 4 millones de peruanos presentan alguna afección mental y solo en Tacna el $22 \%$ de la población ha padecido algún problema de salud mental. Particularmente, en el entorno laboral las personas manifiestan presentar cuadros de estrés, depresión, ansiedad absentismo laboral y pérdida de productividad (2).

En consecuencia, el escenario social actual nos plantea atender esa problemática que debilita el progreso de las instituciones públicas. Es sabido que los sistemas educativos son el soporte de una nación y cumplen un papel fundamental en la formación de los futuros profesionales de las diferentes áreas del saber, los cuales en forma constante siguen un proceso adaptativo hacia los grandes cambios de la sociedad. La universidad, por tanto, plantea la exigencia de analizar y promover una responsabilidad social que cumpla los parámetros éticos y humanos. La salud mental, por otro lado, es un eje transversal en las prioridades de salud por ciclos de vida, además, es definido por la OMS como el estado de bienestar que permite al individuo realizar su potencial, enfrentarse a las dificultades usuales de la vida, trabajar productiva y fructíferamente, así como contribuir con su comunidad (3).

Es por ello que uno de los desafíos de la universidad se relaciona en demostrar que existe efectividad en su funcionamiento y razón de ser, y que se encuentran orientados plenamente para ayudar en la resolución de las necesidades y problemáticas existentes en la sociedad que les rodea. En Tacna, la Universidad Nacional Jorge Basadre Grohmann se presenta como una institución superior pública cuyas políticas de responsabilidad social son recientes, por lo tanto, los actores educativos desconocen la implicancia de ser ciudadano socialmente responsable.

Los actores educativos, al estar en un ambiente académico, están sometidos a altos grados de estrés dado que las cargas horarias muchas veces sobrepasan la capacidad de resistencia física y psíquica. Esto ocasiona problemas de salud y, paulatinamente, una pérdida de interés por el desenvolvimiento de las tareas propias de cada actor educativo (4). Esta investigación plantea conocer la responsabilidad social universitaria y la salud mental positiva del personal administrativo, docentes y estudiantes de la Universidad Nacional Jorge Basadre Grohmann en el año 2018.

\section{Material y métodos}

La presente investigación tiene un enfoque cuantitativo de tipo descriptivo, de diseño no experimental. La población de estudio estuvo constituida por 8361 actores educativos (docentes, administrativos y estudiantes) de las diversas facultades de la Universidad Nacional Jorge Basadre Grohmann de Tacna en el año 2018. Se obtuvo una muestra de 636 actores educativos, los cuales se estratificaron mediante un muestreo no probabilístico por conveniencia de la siguiente manera un $55 \%$ para los estudiantes de un total de 348 , un $25 \%$ para los docentes de un total de 162 y un $20 \%$ para el personal administrativo de un total de 126.

Se utilizó como técnica la encuesta y como instrumento el cuestionario. Se aplicaron dos cuestionarios uno que corresponde al cuestionario de responsabilidad social de los actores educativos (5) y un cuestionario para la salud mental positiva a los actores educativos (6). Así, se aplicó el cuestionario a administrativos, docentes, y estudiantes con 27, 56, y 40 ítems respectivamente. Cada ítem contenía alternativa de respuesta según la escala Likert del 1 al 5, tal como totalmente en desacuerdo, en desacuerdo, ni en desacuerdo ni de acuerdo, de acuerdo y totalmente de acuerdo.

\section{Resultados}

En la Tabla 1 se observa que el $61,1 \%$ de docentes y el 63,5 5 de administrativos se encuentran en la etapa adulto maduro. Por otro lado, el 99,1\% de estudiantes se encuentran en la etapa de adulto joven .Según sexo, el 72,8 \% de docentes es masculino y el 27,2 $\%$ es femenino. Respecto a los estudiantes, el 52,3\% son de sexo masculino y $47,7 \%$ son de sexo femenino. 
Tabla 1. Distribución según etapas de vida y sexo de los actores educativos de la UNJBG, Tacna-2018

\begin{tabular}{|c|c|c|c|c|c|c|}
\hline \multirow[b]{2}{*}{ Características } & \multicolumn{5}{|c|}{ Actor educativo } & istrativos \\
\hline & $\mathrm{n}$ & $\%$ & $\mathrm{n}$ & $\%$ & $\mathrm{n}$ & $\%$ \\
\hline $\begin{array}{l}\text { a) Etapa de vida } \\
\text { Adulto joven } \\
\text { (18-29 años ) }\end{array}$ & 6 & 3,7 & 345 & 99,1 & 23 & 18,3 \\
\hline $\begin{array}{l}\text { Adulto maduro } \\
\text { (30-59 años) }\end{array}$ & 99 & 61,1 & 3 & 0,9 & 80 & 63,5 \\
\hline $\begin{array}{l}\text { Adulto mayor } \\
\text { (> }>00 \text { años })\end{array}$ & 57 & 35,1 & . & - & 23 & 18,3 \\
\hline Total & 162 & 100,0 & 348 & 100,0 & 126 & 100,0 \\
\hline b) Sexo & & & & & & \\
\hline Masculino & 118 & 72,8 & 166 & 47,7 & 40 & 31,7 \\
\hline Femenino & 44 & 27,2 & 182 & 52,3 & 86 & 68,3 \\
\hline Total & 162 & 100,0 & 348 & 100,0 & 126 & 100,0 \\
\hline
\end{tabular}

En la Tabla 2 se puede observar que el $47,6 \%$ de administrativos, el 38,9\% de docentes y el $40,2 \%$ de estudiantes refieren estar ni en desacuerdo ni de acuerdo en procurar reunirse o hablar con alguien para compartir experiencias y asumir la responsabilidad de sus actos y sus decisiones respectivamente.

Tabla 2. Distribución de la responsabilidad social universitaria según la sub dimensión: lucha contra el asistencialismo y paternalismo en el servicio universitario A la comunidad en los actores educativos de la Universidad Nacional Jorge Basadre Grohmann de Tacna en el 2018

\begin{tabular}{|c|c|c|c|c|c|c|c|c|c|c|c|c|c|}
\hline \multirow[t]{2}{*}{$\begin{array}{l}\text { Actor } \\
\text { educativos }\end{array}$} & \multirow[t]{2}{*}{ Ítem } & \multicolumn{2}{|c|}{$\begin{array}{l}\text { Totalmente } \\
\text { desacuerdo }\end{array}$} & \multicolumn{2}{|c|}{$\begin{array}{c}\text { En } \\
\text { desacuerdo }\end{array}$} & \multicolumn{2}{|c|}{$\begin{array}{l}\text { Ni desacuerdo } \\
\text { ni de acuerdo }\end{array}$} & \multicolumn{2}{|c|}{ De acuerdo } & \multicolumn{2}{|c|}{$\begin{array}{l}\text { Totalmentede } \\
\text { acuerdo }\end{array}$} & \multicolumn{2}{|c|}{ Total } \\
\hline & & $\mathrm{n}$ & $\%$ & $\mathrm{n}$ & $\%$ & $\mathrm{n}$ & $\%$ & $\mathrm{n}$ & $\%$ & $\mathrm{n}$ & $\%$ & $\mathrm{n}$ & $\%$ \\
\hline \multirow{3}{*}{ Administrativos } & $\begin{array}{l}\text { Ítem } \\
24\end{array}$ & 14 & 11,1 & 34 & 27 & 42 & 33,3 & 33 & 26,2 & 3 & 2,4 & 126 & 100 \\
\hline & $\begin{array}{l}\text { Ítem } \\
25\end{array}$ & 9 & 7,1 & 25 & 19,8 & 43 & 34,1 & 46 & 36,5 & 3 & 2,4 & 126 & 100 \\
\hline & $\begin{array}{l}\text { Ítem } \\
26\end{array}$ & 4 & 3,2 & 26 & 20,6 & 60 & 47,6 & 30 & 23,8 & 6 & 4,8 & 126 & 100 \\
\hline \multirow{3}{*}{ Docentes } & $\begin{array}{l}\text { Ítem } \\
24\end{array}$ & 19 & 11,7 & 28 & 17,3 & 57 & 35,2 & 38 & 23,5 & 20 & 12,3 & 162 & 100 \\
\hline & $\begin{array}{l}\text { Ítem } \\
25\end{array}$ & 14 & 8,6 & 19 & 11,7 & 39 & 24,1 & 60 & 37 & 30 & 18,5 & 162 & 100 \\
\hline & $\begin{array}{l}\text { Ítem } \\
26\end{array}$ & 16 & 9,9 & 27 & 16,7 & 63 & 38,9 & 35 & 21,6 & 21 & 13,0 & 162 & 100 \\
\hline \multirow{3}{*}{ Estudiantes } & $\begin{array}{l}\text { Ítem } \\
33\end{array}$ & $\begin{array}{l}1 \\
3\end{array}$ & 3,7 & 51 & 14,7 & 140 & 40,2 & 109 & 31,3 & 35 & 10,1 & 348 & 100 \\
\hline & $\begin{array}{l}\text { Ítem } \\
34\end{array}$ & $\begin{array}{l}1 \\
5\end{array}$ & 4,3 & 48 & 13,8 & 119 & 34,2 & 126 & 36,2 & 40 & 11,5 & 348 & 100 \\
\hline & Item & $\begin{array}{l}1 \\
4\end{array}$ & 4,0 & 52 & 14,9 & 135 & 38,8 & 120 & 34,5 & 27 & 7,8 & 348 & 100 \\
\hline
\end{tabular}

En la Tabla 3 se puede observar: que los administrativos en un $44,4 \%$, los docentes en un $35,8 \%$ y los estudiantes en un 40,2\% manifiestan estar ni en desacuerdo, ni de acuerdo en que la universidad busque utilizar sus campañas de marketing para promover valores y temas de responsabilidad social y que los mensajes publicitarios que difunde la universidad sean elaborados con criterios éticos y de responsabilidad social respectivamente; asimismo, el 4,6\% de estudiantes están totalmente en desacuerdo en que la universidad invite a mantener buenas relaciones con las demás universidades con las cuales compite. 
Tabla 3. Distribución de la responsabilidad social universitaria según la sub dimensión comunicación y marketing responsable en los actores educativos de la Universidad Nacional Jorge Basadre Grohmann, Tacna2018

\begin{tabular}{|c|c|c|c|c|c|c|c|c|c|c|c|c|c|}
\hline \multirow[t]{2}{*}{$\begin{array}{l}\text { Actores } \\
\text { educativos }\end{array}$} & \multirow[t]{2}{*}{ Ítems } & \multicolumn{2}{|c|}{$\begin{array}{l}\text { Totalmente } \\
\text { desacuerdo }\end{array}$} & \multicolumn{2}{|c|}{$\begin{array}{c}\text { En } \\
\text { desacuerdo }\end{array}$} & \multicolumn{2}{|c|}{$\begin{array}{c}\mathrm{Ni} \\
\text { desacuerdo ni } \\
\text { de acuerdo }\end{array}$} & \multicolumn{2}{|c|}{ De acuerdo } & \multicolumn{2}{|c|}{$\begin{array}{l}\text { Totalmente } \\
\text { de acuerdo }\end{array}$} & \multicolumn{2}{|c|}{ Total } \\
\hline & & $\mathrm{n}$ & $\%$ & $\mathrm{n}$ & $\%$ & $\mathrm{n}$ & $\%$ & $\mathrm{n}$ & $\%$ & $\mathrm{n}$ & $\%$ & $\mathrm{n}$ & $\%$ \\
\hline \multirow{3}{*}{ Administrativo } & $\begin{array}{l}\text { Item } \\
20\end{array}$ & 8 & 6,3 & 32 & 25,4 & 51 & 40,5 & 31 & 24,6 & 4 & 3,2 & 126 & 100 \\
\hline & $\begin{array}{l}\text { Ítem } \\
21\end{array}$ & 8 & 6,3 & 23 & 18,3 & 56 & 44,4 & 36 & 28,6 & 3 & 2,4 & 126 & 100 \\
\hline & $\begin{array}{c}\text { Ítem } \\
19\end{array}$ & 21 & 13 & 34 & 21 & 43 & 26,5 & 39 & 24,1 & 25 & 15,4 & 162 & 100 \\
\hline \multirow[t]{2}{*}{ Docente } & $\begin{array}{l}\text { Ítem } \\
20\end{array}$ & 5 & 3,1 & 19 & 11,7 & 45 & 27,8 & 53 & 32,7 & 40 & 24,7 & 162 & 100 \\
\hline & $\begin{array}{c}\text { Ítem } \\
21\end{array}$ & 24 & 14,8 & 27 & 16,7 & 58 & 35,8 & 35 & 21,6 & 18 & 11,1 & 162 & 100 \\
\hline \multirow{4}{*}{ Estudiantes } & $\begin{array}{c}\text { Ítem } \\
17\end{array}$ & 19 & 5,5 & 50 & 14,4 & 139 & 39,9 & 110 & 31,6 & 30 & 8,6 & 348 & 100 \\
\hline & $\begin{array}{c}\text { Ítem } \\
18\end{array}$ & 13 & 3,7 & 35 & 10,1 & 128 & 36,8 & 140 & 40,2 & 32 & 9,2 & 348 & 100 \\
\hline & $\begin{array}{c}\text { Ítem } \\
19\end{array}$ & 16 & 4,6 & 29 & 8,3 & 130 & 37,4 & 135 & 38,8 & 38 & 10,9 & 348 & 100 \\
\hline & $\begin{array}{l}\text { Ítem } \\
20\end{array}$ & 19 & 5,5 & 46 & 13,2 & 133 & 38,2 & 120 & 34,5 & 30 & 8,6 & 348 & 100 \\
\hline
\end{tabular}

En la tabla 4 se puede observar que el $73 \%$ de administrativos y el $81,5 \%$ de docentes manifiestan que siempre asumen la responsabilidad de sus actos y sus decisiones. Por otro lado, el $50 \%$ de estudiantes manifiestan que casi siempre pueden ponerse en el lugar de los (las) demás y comprenderlos (las), así como de poder expresar sus sentimientos y opiniones ante las personas sin faltarles el respeto.

Tabla 4. Distribución de la salud mental positiva según la subdimensión de habilidades cognitivas y emotivas de imaginar que se forma parte de la sociedad y de cómo se sienten las personas en los actores educativos de la Universidad Nacional Jorge Basadre Grohmann de Tacna-2018

\begin{tabular}{|c|c|c|c|c|c|c|c|c|c|c|c|c|c|}
\hline \multirow[t]{2}{*}{$\begin{array}{c}\text { Actores } \\
\text { educativos }\end{array}$} & \multirow[t]{2}{*}{ Ítems } & \multicolumn{2}{|c|}{ Nunca } & \multicolumn{2}{|c|}{$\begin{array}{c}\text { Casi } \\
\text { Nunca }\end{array}$} & \multicolumn{4}{|c|}{$\begin{array}{cc}\text { Escala valorativa } & \\
\text { Algunas } & \text { Casi } \\
\text { Veces } & \text { Siempre }\end{array}$} & \multicolumn{2}{|c|}{ Siempre } & \multicolumn{2}{|c|}{ Total } \\
\hline & & $\mathrm{n}$ & $\%$ & $\mathrm{n}$ & $\%$ & $\mathrm{n}$ & $\%$ & $\mathrm{n}$ & $\%$ & $\mathrm{n}$ & $\%$ & $\mathrm{n}$ & $\%$ \\
\hline \multirow{7}{*}{ Administrativo } & $\begin{array}{c}\text { Ítem } \\
31\end{array}$ & 1 & 0,6 & 1 & 0,6 & 3 & 1,9 & 37 & 22,8 & 120 & 74,1 & 126 & 100 \\
\hline & $\begin{array}{l}\text { Ítem } \\
32\end{array}$ & 2 & 1,2 & - & - & 14 & 8,6 & 57 & 35,2 & 89 & 54,2 & 126 & 100 \\
\hline & $\begin{array}{l}\text { Ítem } \\
33\end{array}$ & 1 & 0,6 & - & - & 5 & 3,1 & 46 & 28,4 & 110 & 67,9 & 126 & 100 \\
\hline & $\begin{array}{c}\text { Ítem } \\
34\end{array}$ & - & - & - & - & 4 & 2,5 & 26 & 16 & 132 & 81,5 & 126 & 100 \\
\hline & $\begin{array}{l}\text { Ítem } \\
35\end{array}$ & - & - & - & - & 7 & 4,3 & 40 & 24,7 & 115 & 71 & 126 & 100 \\
\hline & $\begin{array}{c}\text { Ítem } \\
36\end{array}$ & - & - & - & - & 6 & 3,7 & 61 & 37,7 & 95 & & 126 & 100 \\
\hline & $\begin{array}{c}\text { Ítem } \\
37\end{array}$ & - & - & 1 & 0,6 & 5 & 3,1 & 57 & 35,2 & 99 & 61,1 & 126 & 100 \\
\hline
\end{tabular}




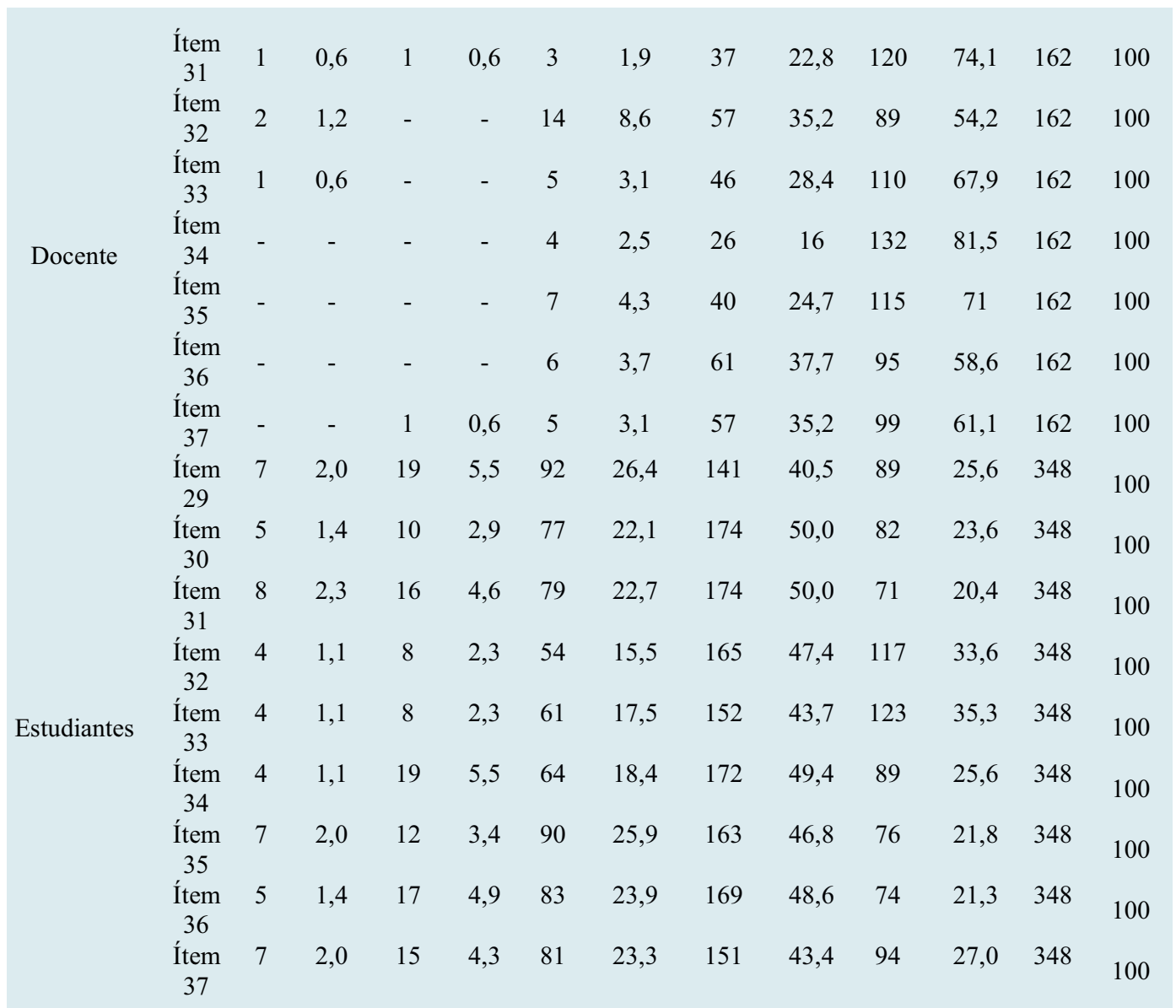

En la tabla 5, se puede observar que el 45,7\% de docentes manifiestan estar ni en desacuerdo ni de acuerdo en que la universidad cuente con dispositivos regulares para el seguimiento de las políticas públicas, así como de la identificación y del análisis de los grandes temas de la sociedad. Además, un el 3,7\% manifiesta estar totalmente en desacuerdo y en desacuerdo en que los estudiantes de pregrado deben obligatoriamente practicar la investigación formativa en sus asignaturas académicas.

Tabla 5. Distribución de la responsabilidad social universitaria según la sub dimensión comunicación y marketing responsable en los actores educativos de la Universidad Nacional Jorge Basadre Grohmann, Tacna2018

\begin{tabular}{|c|c|c|c|c|c|c|c|c|c|c|c|c|c|}
\hline \multirow{3}{*}{ Actor } & \multirow{3}{*}{ Ítems } & \multicolumn{10}{|c|}{ Escala valorativa } & \multirow{2}{*}{\multicolumn{2}{|c|}{ Total }} \\
\hline & & \multicolumn{2}{|c|}{$\begin{array}{l}\text { Totalmente } \\
\text { desacuerdo }\end{array}$} & \multicolumn{2}{|c|}{$\begin{array}{c}\text { En } \\
\text { desacuerdo }\end{array}$} & \multicolumn{2}{|c|}{$\begin{array}{l}\text { Ni desacuerdo } \\
\text { ni de acuerdo }\end{array}$} & \multicolumn{2}{|c|}{$\begin{array}{c}\text { De } \\
\text { acuerdo }\end{array}$} & \multicolumn{2}{|c|}{$\begin{array}{l}\text { Totalmente } \\
\text { de acuerdo }\end{array}$} & & \\
\hline & & $\mathrm{n}$ & $\%$ & $\mathrm{n}$ & $\%$ & $\mathrm{n}$ & $\%$ & $\mathrm{n}$ & $\%$ & $\mathrm{n}$ & $\%$ & $\mathrm{n}$ & $\%$ \\
\hline \multirow{12}{*}{ Docentes } & Ítem 45 & 15 & 9,3 & 21 & 13 & 47 & 29 & 35 & 21,6 & 44 & 27,2 & 162 & 100 \\
\hline & Item 46 & 12 & 7,4 & 22 & 13,6 & 60 & 37 & 37 & 22,8 & 31 & 19,1 & 162 & 100 \\
\hline & Ítem 47 & 20 & 12,3 & 22 & 13,6 & 74 & 45,7 & 33 & 20,4 & 13 & 8 & 162 & 100 \\
\hline & Ítem 48 & 35 & 21,6 & 26 & 16,0 & 51 & 31,5 & 26 & 16 & 24 & 14,8 & 162 & 100 \\
\hline & Ítem 49 & 23 & 14,2 & 30 & 18,5 & 42 & 25,9 & 36 & 22,2 & 31 & 19,1 & 162 & 100 \\
\hline & Ítem 50 & 14 & 8,6 & 28 & 17,3 & 45 & 27,8 & 44 & 27,2 & 31 & 19,1 & 162 & 100 \\
\hline & Ítem 51 & 20 & 12,3 & 25 & 15,4 & 65 & 40,1 & 37 & 22,8 & 15 & 9,3 & 162 & 100 \\
\hline & Item 52 & 21 & 13 & 20 & 12,3 & 65 & 40,1 & 40 & 24,7 & 16 & 9,9 & 162 & 100 \\
\hline & Item 53 & 15 & 9,3 & 23 & 14,2 & 64 & 39,5 & 43 & 26,5 & 17 & 10,5 & 162 & 100 \\
\hline & Ítem 54 & 6 & 3,7 & 6 & 3,7 & 29 & 17,9 & 52 & 32,1 & 69 & 42,6 & 162 & 100 \\
\hline & Item 55 & 13 & 8 & 14 & 8,6 & 39 & 24,1 & 45 & 27,8 & 51 & 31,5 & 162 & 100 \\
\hline & Ítem 56 & 19 & 11,7 & 14 & 8,6 & 45 & 27,8 & 43 & 26,5 & 41 & 25,3 & 162 & 100 \\
\hline
\end{tabular}


En la Tabla 6 se puede observar que el 51,6\% de administrativos, y el $61,1 \%$ de docentes manifiestan nunca haber perdido interés en las cosas. Por otro lado, el 34,8 \% de estudiantes, manifiestan que algunas veces pierden el contacto con la realidad.

Tabla 6. Distribución de la salud mental positiva según la sub dimensión de componente cognitivo de distorsión y percepción de malestar físico y mental en los actores educativos de la Universidad Nacional Jorge Basadre Grohmann, Tacna-2018

\begin{tabular}{|c|c|c|c|c|c|c|c|c|c|c|c|c|c|}
\hline \multirow[t]{2}{*}{$\begin{array}{l}\text { Actores } \\
\text { educativos }\end{array}$} & Ítems & & nca & \multicolumn{4}{|c|}{$\begin{array}{cc} & \text { Escala valorativa } \\
\text { Casi nunca } & \text { Algunas } \\
\text { veces }\end{array}$} & \multicolumn{2}{|c|}{$\begin{array}{c}\text { Casi } \\
\text { siempre }\end{array}$} & \multicolumn{2}{|c|}{ Siempre } & \multicolumn{2}{|c|}{ Total } \\
\hline & & $\mathrm{n}$ & $\%$ & $\mathrm{n}$ & $\%$ & $\mathrm{n}$ & $\%$ & $\mathrm{n}$ & $\%$ & $\mathrm{n}$ & $\%$ & $\mathrm{n}$ & $\%$ \\
\hline \multirow{4}{*}{ 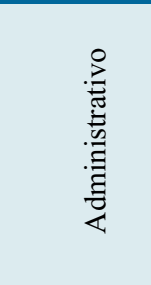 } & $\begin{array}{l}\text { Item } \\
51\end{array}$ & 2 & 1,6 & 9 & 7,1 & 48 & 38,1 & 34 & 27 & 33 & 26,2 & 126 & 100 \\
\hline & $\begin{array}{l}\text { Ítem } \\
52\end{array}$ & 49 & 38,9 & & 31,7 & 28 & 22,2 & 4 & 3,2 & 5 & 4,0 & 126 & 100 \\
\hline & $\begin{array}{c}\text { Ítem } \\
53\end{array}$ & 46 & 36,5 & 52 & 41,3 & 23 & 18,3 & 5 & 4 & 0 & 0 & 126 & 100 \\
\hline & $\begin{array}{l}\text { Ítem } \\
54\end{array}$ & 65 & 51,6 & 36 & 28,6 & 21 & 16,7 & 4 & 3,2 & 0 & 0 & 126 & 100 \\
\hline \multirow{4}{*}{ 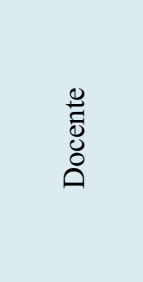 } & 51 & 55 & 34 & 37 & 22,8 & 47 & 29 & 10 & 6,2 & 13 & 8 & 162 & 100 \\
\hline & $\begin{array}{l}\text { Ítem } \\
52\end{array}$ & 74 & 45,7 & 43 & 26,5 & 30 & 18,5 & 7 & 4,3 & 8 & 4,9 & 162 & 100 \\
\hline & $\begin{array}{c}\text { Ítem } \\
53\end{array}$ & 75 & 46,3 & 58 & 35,8 & 30 & 12,3 & 6 & 3,7 & 3 & 1,9 & 162 & 100 \\
\hline & $\begin{array}{c}\text { Ítem } \\
54\end{array}$ & 99 & 61,1 & 41 & 25,3 & 15 & 9,3 & 3 & 1,9 & 4 & 2,5 & 162 & 100 \\
\hline \multirow{6}{*}{ 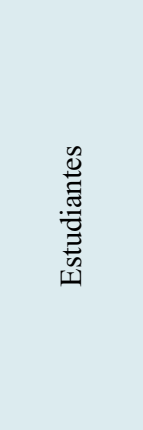 } & $\begin{array}{l}\text { Ítem } \\
50\end{array}$ & 59 & 17,0 & 68 & 19,5 & 105 & 30,2 & 79 & 22,7 & 37 & 10,6 & 348 & 100 \\
\hline & $\begin{array}{l}\text { Ítem } \\
51\end{array}$ & 35 & 10,1 & 72 & 20,7 & 114 & 32,8 & 81 & 23,3 & 46 & 13,2 & 348 & 100 \\
\hline & $\begin{array}{l}\text { Ítem } \\
52\end{array}$ & 65 & 18,7 & 73 & 21,0 & 121 & 34,8 & 58 & 16,7 & 31 & 8,9 & 348 & 100 \\
\hline & $\begin{array}{c}\text { Ítem } \\
53\end{array}$ & 65 & 18,7 & 75 & 21,6 & 112 & 32,2 & 68 & 19,5 & 28 & 8,0 & 348 & 100 \\
\hline & $\begin{array}{c}\text { Ítem } \\
54\end{array}$ & 47 & 13,5 & 78 & 22,4 & 99 & 28,4 & 85 & 24,4 & 39 & 11,2 & 348 & 100 \\
\hline & $\begin{array}{c}\text { Ítem } \\
55\end{array}$ & 76 & 21,8 & 79 & 22,7 & 116 & 33,3 & 56 & 16,1 & 21 & 6,0 & 348 & 100 \\
\hline
\end{tabular}

\section{Discusión}

En la tabla de características de etapas de vida, se registra que los docentes y administrativos son adultos mayores y los estudiantes adultos jóvenes. Estos resultados se contrastan con Aldrete et al. (7), que en un estudio titulado "Condiciones de trabajo y salud de los docentes de enseñanza media superior de una universidad pública" (2016) señalan que el rango promedio de los docentes fue de 24 a 70 años. También, este trabajo coinciden con los resultados encontrados por Collado et al. (12), quienes en un estudio titulado "condiciones de trabajo y salud mental en docentes universitarios y de enseñanza media de Mendoza, Argentina: Entre el compromiso y el desgaste emocional" (2016) encontraron que los docentes tenían entre 27 a 67 años de edad; además que el $40 \%$ de este plantel poseía más de 55 años. En consonancia, Chau y
Saravia (8), en su informe de proyecto de universidades saludables en el año 2012, señala que el rango de edad de los estudiantes estuvo entre los 16 y 22 años, correspondientes a la etapa de adulto joven (8). Por lo señalado anteriormente, se deduce que los actores educativos son parte de una población institucional que tiene un crecimiento en cada uno de los estratos de vida, observándose que los docentes y administrativos se encuentran en la etapa adultos maduros; es decir entre los 35 a 65 años de edad. Por otro lado, los estudiantes se encuentran en la etapa adulto joven estando; es decir entre los 18 y 29 años de edad.

Las subdimensiones de la variable responsabilidad social de los actores educativos presentan una diversidad de porcentajes resaltando la subdivisión lucha contra el asistencialismo y paternalismo en el servicio universitario a la comunidad en los actores educativos de la Universidad Nacional 
Jorge Basadre Grohmann. Contrastando, con Aguirre y Martinez (9), que en su investigación, "La responsabilidad social universitaria en los docentes y no docentes" en una universidad de México (2014), obtuvieron como resultados que el $74,4 \%$ de los docentes tienen conocimiento y ha percibido que es la responsabilidad social universitaria; por otro lado, el $27,3 \%$ del personal no docente -a pesar de conocer la RSU- no han sido participes ni lo han percibido; además, el resto de no docentes $72,7 \%$ no conocían este concepto. Asimismo, se defiere con Gómez et al.(13), quienes en su estudio "Practicando la responsabilidad social universitaria en el Caribe: perspectivas de los público internos", obtuvieron como resultados que los diversos públicos internos están conscientes de lo que significa en la teoría y en la práctica ser una universidad socialmente responsable; aunque, también, reconocen oportunidades de mejora y desafíos futuros.

A partir de lo descrito anteriormente, se concluye que los resultados hallados sobre la percepción social universitaria y sus sub dimensiones: lucha contra el asistencialismo y paternalismo en el servicio universitario a la comunidad en los actores educativos y comunicación y marketing responsable en los actores educativos, entre otros, nos muestran que los actores educativos muestran indiferencia y se mantiene al margen; es decir, no tienen un compromiso consciente ni un trabajo articulado con los esfuerzos de la UNJBG para proyectarse a la sociedad según las políticas de gestión institucional como el RSU para el desarrollo humano sostenible. Es merecedor resaltar que la comunidad universitaria es un ente social conformado por los actores educativos quienes participan en el que hacer universitario; en otras palabras, en sus funciones y responsabilidades.

Por otro lado, los hallazgos de la salud mental positiva de los actores educativos muestran en su gran mayoría incidencia en un siempre, casi siempre, algunas veces y nunca en las sub dimensiones habilidades cognitivas y emotivas de imaginar que se forma parte de la sociedad y de cómo se sienten las personas. En los actores educativos y componente cognitivo de distorsión y percepción de malestar físico y mental en los actores educativos, los resultados obtenidos difieren con Vázquez et al. (10), quienes en un estudio sobre "La salud mental positiva ocupacional en profesores universitarios" (2016), obtuvieron que la dimensión espiritual destaca en un nivel muy alto con un $47 \%$; en tercer lugar, se encontraba la dimensión socio afectiva con un $26 \%$ en nivel muy alto y; por último, la dimensión comportamental, presente en el menor porcentaje en nivel muy alto. Esto contrasta con Bobadilla (11), en su estudio sobre "auditoria social y su influencia en la responsabilidad social universitaria de las universidades públicas del CRISUR, 2015", quien concluyo que la universidad Jorge Basadre Grohmann tiene un nivel de cumplimiento medio (17,32\%) de la responsabilidad social universitaria. Así también, se contrasta con Gauna (14) en su estudio "La responsabilidad social de la universidad y la salud mental de los estudiantes universitarios", quien concluyó que la universidad de Buenos Aires, mediante extensión universitaria, debe y puede promover actividades que contribuyan a la salud mental de su población estudiantil, contando con la capacidad de sus recursos humanos para generar conocimiento, propuestas y recursos de gestión. Entonces, podemos concluir que la salud mental positiva de los actores educativos tanto en docentes como administrativos tiene una percepción regular.

\section{Conclusiones}

- La concepción de la responsabilidad social universitaria entre el personal administrativo, docentes y estudiantes como actores educativos son siempre, casi siempre y ni en de acuerdo ni en desacuerdo.

- Existe diferencia significativa en la concepción de la salud mental positiva de los actores educativos.

- La concepción de la salud mental entre el personal administrativo, docentes y estudiantes como actores educativos son siempre, casi siempre, algunas veces y nunca.

\section{Limitaciones}

- Se percibe debilidad para el estudio, ya que docentes, administrativos y estudiantes no manifiestan tener conocimientos sobre responsabilidad social.

- No se contó con las facilidades para la realización de la encuesta y el permiso respectivo para el ingreso en las distintas facultades.

- La negativa de muchos docentes, administrativos y estudiantes para la realización de la encuesta.

- No existen estudios de investigación relacionados con la problemática planteada en Tacna. 


\section{Referencias bibliográficas}

1. Ley Universitaria en el Perú 30220

2. Ministerio de Salud: Lineamientos de Políticas Sectoriales es en Salud Mental. Lima: SINCO Diseño; 2018.

3. Revista Iberoamericana de Diagnóstico y EvaluaciónMinisterio de Salud. Modelo de Abordaje de Promoción de la Salud en el Perú. Lima. Gobierno del Perú 2005

4. Tapia, N. "Calidad académica y responsabilidad social: el aprendizaje servicio como puente entre dos culturas universitarias", en Martínez, M. (ed.). Aprendizaje Servicio y Responsabilidad Social de las universidades. Barcelona: Octaedro-ICE.2010.

5. Vallaeys, F.Cruz, C.Sasia, P. Responsabilidad social Universitaria: Manual de primeros pasos.Oficina de Relaciones Externas del BID.2009

6. Barrera G.Lolbe M, Flores G et al. Construcción de una escala de salud mental positiva para adultos en una población mexicana. Revista Iberoamericana de Diagnóstico y Evaluación, 2015; 1(39):22-23.

7. Aldrete M., González R., Leon S., Contreras M., Hidalgo G., Pérez J. y Galván M. Condiciones de trabajo y salud de los docentes de enseñanza media superior de una universidad pública. Rev. Educación y desarrollo. México, 2016.

8. Chau C,Saravia J. Conductas de Salud en Estudiantes Universitarios Limeños: Validación del CEVJU . Revista Iberoamericana de Diagnóstico y Evaluación - e Avaliação Psicológica. RIDEP, 2016; 41(1): 90-103.

9. Aguirre M. Martínez P. Magaña K. La percepción de la responsabilidad social universitaria en los docentes y no docentes. En: XIX Congreso internacional de investigación en ciencias administrativas. Gestión de las organizaciones rumbo al 3er milenio "De la regionalización a la globalización". México: Universidad Veracruzana; 2014.

10. Vázquez Pando M et, al. . Salud Mental Positiva Ocupacional: propuesta de un modelo teórico para el abordaje positivo de la salud mental en el trabajo, Saúde Soc. São Paulo, 2017; 26 (2): 584-595.

11. Bobadilla M. Auditoría social y su influencia en la responsabilidad social universitaria de las universidades públicas del Crisur, 2015. REVISTA CIENCIA Y TECNOLOGÍA Para el DesarrolloUJCM 2017; 3(5):63-70.

12. Collado P, Soria C, Canafoglia et al. Condiciones de trabajo y salud en docentes universitaria y de enseñanza media de Mendoza, argentina: entre el compromiso y el desgaste emocional Salud. Salud Colectiva, Universidad Nacional de Lanus, Issn $1669-2381$, e is S N 1851 8265,doi:10.18294/sc.2016.710

13. Practicando la Responsabilidad Social Universitaria en el Caribe: Perspectivas de los públicos internos. Rev. Digit. Invest. Docencia Univ. [online], 2018;

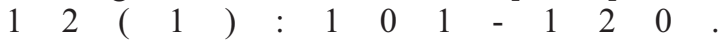
http://dx.doi.org/10.19083/ridu.12.714

14. Gauna, María. La responsabilidad social de la universidad y la salud mental de los estudiantes universitarios. XIV Jornadas de Investigación y Tercer Encuentro de Investigadores en Psicología del Mercosur. Facultad de Psicología - Universidad de Buenos Aires, Buenos Aires; 2007.

\section{Correspondencia}

nvp1712@yahoo.com.mx
Fecha de recepción: 12 de julio de 2019

Fecha de aceptación: 05 de noviembre de 2019 